\title{
Factors Associated with Indeterminate and False Negative Results of QuantiFERON-TB Gold In-Tube Test in Active Tuberculosis
}

Kiwon Cho, M.D., Eunha Cho, M.D., Soohoon Kwon, M.D., Sanghyuk Im, M.D., In Sohn, M.D., Sookhee Song, M.D., Hyeok Kim, Ph.D., Suhyun Kim, M.D.

Department of Internal Medicine, Seoul Medical Center, Seoul, Korea

Background: The sensitivities and specificities of interferon-gamma release assays (IGRAs) vary among different population studies, and the data on the routine use of IGRAs are limited. The aim of this study was to evaluate the role of QuantiFERON-TB Gold In-Tube (QFT-GIT) test in the diagnosis of active tuberculosis.

Methods: We conducted a prospective study, enrolling 77 patients with suspected pulmonary tuberculosis (TB), at a secondary care teaching hospital in Seoul.

Results: In total, $12(15.6 \%)$ patients showed indeterminate results due to positive control failure on the QFT-GIT test. Indeterminate results were significantly associated with the elderly, history of the intensive care unit stay, lymphocytopenia, especially low CD4 count, increased C-reactive protein and decreased protein levels. Of the 77 patients, $44(57.1 \%)$ were diagnosed with active pulmonary tuberculosis, and the percentage of false negative results of the QFT-GIT was $36.4 \%$ (vs. 31.8\% with TST). In the TB group with $>65$ years old ( $\mathrm{n}=12$ ), the proportions of the indeterminate $(33.3 \%$ vs. 3.1\%) and the false negative results (58.3\% vs. 25.0\%) of the QFT-GIT were significantly higher than in the younger TB group $(n=32)$.

Conclusion: Indeterminate and false negative results of QFT-GIT test were not infrequent in tuberculosis, especially in the elderly. Care should be considered for the interpretation with the elderly, immunocompromised, chronic and severely diseased patients.

Key Words: Interferon-gamma Release Tests; Tuberculin Test; Aged

\section{Introduction}

Acid-fast bacilli (AFB) staining of sputum has a sensitivity of only $50 \sim 60 \%$, and cultures for tuberculosis (TB) diagnosis usually require several weeks for results. Moreover, diagnosis and treatment decisions may be difficult in case with clinical suspicion of TB and negative AFB sputum smears. In this case, the rapid diagnosis of TB classically relies on the results of different methods, including the tuberculin skin test (TST), chest radiography, nucleic acid amplification (NAA) test of

Address for correspondence: Suhyun Kim, M.D.

Department of Internal Medicine, Seoul Medical Center, 156, Sinnae-ro, Jungnang-gu, Seoul 135-740, Korea

Phone: 82-2-2276-7808, Fax: 82-2-2276-7820

E-mail: sammy7597@naver.com

Received: Jan. 11, 2012

Revised: Jan. 27, 2012

Accepted: Mar. 16, 2012
Mycobacterium tuberculosis (M.TB) and/or pathological examinations, although TST has a disadvantage of poor specificity in bacille Calmette-Guerin (BCG) vaccinated individuals and in those living in high TB prevalence countries ${ }^{1}$. Meanwhile, interferon-gamma release assays (IGRAs), what measure and interpret interferon-gamma (IFN- $\gamma$ ) that is produced by immune responses to early secreted antigenic target 6 (ESAT-6) and culture filtrate protein 10 (CFP-10), has advantages of higher specificity than TST, no need to revisit the hospital after the test, and no boosted effect in a repeated conduct ${ }^{2}$. Since the Food and Drug Administration (FDA) announced that QuantiFERON-TB GOLD test (QFT-G; Cellestis Ltd., Carnegie, Victoria, Australia) can be alternatively used for TB diagnosis instead of TST, QuantiFERON-TB Gold In-Tube test (QFT-GIT; Cellestis Ltd.) and T-SPOT. TB test (Oxford Immunotec, Abingdon, UK) have been pro- 
ven to be used as supportive tests along with other tests for the diagnosis of active pulmonary $\mathrm{TB}$ as well as latent TB infection (LTBI) $)^{3,4}$.

QFT-GIT, which has been developed to improve the limitation of QFT-G that should be performed within several hours after blood collection, enables direct test using a special tubes containing antigens without pipetting or handling, and its sensitivity has been improved due to the addition of TB7.7 to TB antigens ${ }^{4}$. The rate of indeterminate results, significantly associated with elderly and immunocompromised patients, has been reported to be 5 40\% in QFT-G, thereby causing clinical difficulty in the interpretation of test results. Whereas, such high rate of indeterminate results in QFT-G is anticipated to decrease in QFT-GIT. However, there have been few studies performing a detailed investigation of indeterminate results with clinical effectiveness in QFT-GIT with active TB patients ${ }^{5}$. In addition, in the case of elderly patients with $\mathrm{TB}$, the positive rate of $\mathrm{AFB}$ smears is even lower due to difficulty in appropriate expectoration of sputum. Thus, a supportive method is required to improve diagnostic yield in elderly patients ${ }^{6}$, but the results of IGRAs should be carefully interpreted due to decrease in the immunologic status associated with aging ${ }^{7}$. In Korea, studies on the interpretation of the results of QFT performed on elderly TB patients have been insufficiently conducted so far. Therefore, we evaluated the risk factors including age distribution that can influence indeterminate and false negative results when using the QFT-GIT in routine clinical practice in a single institution.

\section{Materials and Methods}

\section{Subjects}

We conducted prospective study after the approval of the Institutional Review Board (IRB) of Seoul Medical Center. Overall 77 patients, who signed the informed consent form among patients with suspected TB underwent QFT-GIT were recruited in the Division of Pulmonology at Seoul Medical Center from January 2010 to August 2010. The presence of active pulmonary TB was defined as identification of M.TB in sputum culture or positive NAA test of M.TB and/or abnormalities suggestive of TB on chest radiography and/or chest computed tomography (CT) scans. The diagnosis of pulmonary $\mathrm{TB}$ in individuals who were culture-negative was based on chest radiography and/or CT scans findings and the clinician's opinion based on clinical manifestations. Patients with culture-negative pulmonary TB showed good clinical and radiographic responses to anti-TB treatment during follow-up. Fibrotic TB sequelae were defined as 'nodulostreaky' lesions in the upper lobes that had not changed for at least 3 months, with negative culture findings in sputum ${ }^{8}$. TB lymphadenitis was diagnosed in the case of caseating granuloma in the lymph node tissues and patients with lymphocyte-predominant exudates pleural effusion showing adenosine deaminase $>40 \mathrm{IU} / \mathrm{L}$ were diagnosed with TB pleurisy. Patients with bacterial pneumonia, non-tuberculous mycobacteria (NTM) lung diseases, lung cancer, fibrotic TB sequelae, and aspergillosis were included in the control group.

\section{Methods}

AFB smear tests or cultures using the sputum or bronchial washing fluid, and NAA test of M.TB were performed on patients with suspected pulmonary TB or TB pleurisy. If the patients had pleural effusion, they underwent pleural fluid analysis and cytologic examinations. QFT-GIT was conducted on all the subjects and TST, CD4/CD8 count, human immunodeficiency virus antibody, complete blood count, blood chemistry, C-reactive protein (CRP) and erythrocyte sedimentation rate measurement were also done. In addition, demographic and clinical data were collected which included age, gender, underlying diseases and history of previous TB disease and/or contact with TB patients, homeless, smoking and receiving immunosuppressive drugs.

1) Tuberculin skin test: The TST was typically performed on the volar side of the forearm using the Mantoux method with 2 tuberculin units of purified protein derivatives (RT23; Statens Serum Institute, Copenhagen, Denmark). Induration size was measured after 
K Cho et al: QuantiFERON-TB Gold In-Tube test in elderly

Table 1. Interpretation criteria of QuantiFERON-TB Gold In-Tube test

\begin{tabular}{lllc}
\hline Interpretation & Nil & \multicolumn{1}{c}{ TB response } & Mitogen response \\
\hline Positive & $\leq 8.0$ & $\geq 0.35 \mathrm{IU} / \mathrm{mL}$ and $\geq 0.25 \%$ of Nil & Any \\
Negative & $\leq 8.0$ & $<0.35 \mathrm{IU} / \mathrm{mL}$ or $<0.25 \%$ of Nil & $\geq 0.5$ \\
Indeterminate & $\leq 8.0$ & $<0.35 \mathrm{IU} / \mathrm{mL}$ or $<0.25 \%$ of Nil & $<0.5$ \\
& $>8.0$ & Any & Any \\
\hline
\end{tabular}

Source: Based on Cellestis Limited. Available from: http://www.cellestis.com/IRM/content/pdf/Quantiferon\%20US\%20VerG-Jan2010\% 20NO\%20TRIMS/pdf ${ }^{4}$.

48 72 hours and we used a 10-mm induration as the positive cut-off value for the TST. History of BCG vaccination was investigated as follows.

2) QuantiFERON-TB Gold In-Tube test: As for the QFT-GIT test, the negative control antigen (Nil), positive control antigen (Mitogen), and TB-specific antigens (ESAT-6, CFP-10, TB7.7 synthetic peptide) were put into a tube treated with heparin, respectively, and then $1 \mathrm{~mL}$ of whole blood was added to each tube, followed by culturing at $37^{\circ} \mathrm{C}$ for approximately $16 \sim 24$ hours. Subsequently, the produced IFN- $\gamma$ was measured via enzyme-linked immunosorbent assay (ELISA) according to the guidelines proposed by the Centers for Disease Control and Prevention (CDC).

If the IFN- $\gamma$ value $\geq 0.35 \mathrm{IU} / \mathrm{mL}$ and $\geq 25 \%$ of the negative control antigen regardless of the value of the positive control antigen, the result of QFT-GIT was adjudged positive. If the IFN- $\gamma$ value of the positive control antigen $\geq 0.5 \mathrm{IU} / \mathrm{mL}$, and the IFN- $\gamma$ value $<0.35$ $\mathrm{IU} / \mathrm{mL}$ or $\leq 25 \%$ of the negative control antigen, the result of QFT-GIT was adjudged negative. For samples where the IFN- $\gamma$ response to Mitogen was less than $0.5 \mathrm{IU} / \mathrm{mL}$ above that for the Nil control, or the IFN- $\gamma$ response to mitogen was greater than $8 \mathrm{IU} / \mathrm{mL}$ above that for the Nil control, the result was deemed indeterminate results (Table 1).

3) Data analysis: As for data, continuous variables were expressed as inter-quartile range (IQR) and categorical variables were expressed as frequency. Fisher's exact test and Mann-Whitney test were calculated for the comparison of clinical features between the groups according to the variables. If $\mathrm{p}$-value $<0.05$, it was con- sidered statistically significant. Analysis was performed using SPSS version 14.0 (SPSS Inc., Chicago, IL, USA).

\section{Results}

This study was performed on 77 patients with clinically suspected active TB, of whom 44 patients were confirmed to have active TB (34 patients with culture-proven TB, 24 patients with positive NAA test of M.TB). The confirmed TB patients consisted of 39 patients with pulmonary TB, 1 patient with miliary TB, 3 patients with $\mathrm{TB}$ pleurisy, and 1 patient with $\mathrm{TB}$ lymphadenitis. The 33 control group consisted of $20 \mathrm{pa}-$ tients with bacterial pneumonia, 3 patients with NTM lung disease, 1 patient with lung cancer, and 9 patients with fibrotic $\mathrm{TB}$ sequelae. The median age of the $\mathrm{TB}$ group was shown to be 48.5 (IQR, 41.0 66.0), and their male to female ratio was shown to be 31 (70.5\%):13 (29.5\%). Meanwhile, the median age of the control group was shown to be 62.0 (IQR, 50.5 70.5), and their male to female ratio was shown to be 22 (66.7\%):11 (33.3\%). No difference in the age and gender was found between the TB and control groups (Table 2).

The result of QFT-GIT was shown to be positive in 23 cases (52.3\%), negative in 16 cases (36.4\%), and indeterminate in 5 cases (11.4\%) in the TB group, whereas the result of TST was shown to be positive $(>10$ $\mathrm{mm})$ in 26 cases $(59.1 \%)$, and negative in 14 cases $(31.8 \%)$. Meanwhile, the result of QFT-GIT was shown to be positive in 7 cases (21.2\%), negative in 19 cases (57.6\%), and indeterminate in 7 cases (21.2\%) in the 
Tuberculosis and Respiratory Diseases Vol. 72. No. 5, May 2012

Table 2. Clinical characteristics and results of QFT-GIT of 77 patients suspected of active pulmonary TB

\begin{tabular}{|c|c|c|c|}
\hline & & TB group $(n=44)$ & Non-TB group $(n=33)$ \\
\hline \multicolumn{2}{|c|}{ Median age, yr (IQR) } & $48.5(41.0 \sim 68.3)$ & $62.0(50.5 \sim 70.5)$ \\
\hline \multicolumn{2}{|c|}{ Gender, M:F } & $31: 13$ & $22: 11$ \\
\hline \multicolumn{2}{|c|}{ Received TB treatment, $\mathrm{n}$} & 13 & 6 \\
\hline \multirow{6}{*}{\multicolumn{2}{|c|}{ Final diagnosis }} & Pulmonary TB (40) & Bacterial pneumonia (20) \\
\hline & & M.TB culture $(+) 35$ & Fibrotic TB sequelae (6) \\
\hline & & M.TB culture $(-) 5$ & NTM lung disease (3) \\
\hline & & TB pleurisy (2) & Lung cancer (1) \\
\hline & & TB lymphadenitis (1) & Others (3) \\
\hline & & Miliary TB (1) & \\
\hline \multirow[t]{3}{*}{ QTF-GIT, n (\%) } & Positive & 23 (52.3) & 7 (21.2) \\
\hline & Negative & $16(36.4)$ & $19(57.6)$ \\
\hline & Indeterminate & $5(11.4)$ & $7(21.2)$ \\
\hline \multirow[t]{2}{*}{ TST, n (\%) } & Positive & $26(59.1)$ & $8(24.2)$ \\
\hline & Negative & $14(31.8)$ & $22(66.7)$ \\
\hline
\end{tabular}

QFT-GIT: QuantiFERON-TB Gold In-Tube; TB: tuberculosis; IQR: inter-quartile range; M: male; F: female; M.TB: Mycobacterium tuberculosis; NTM: non-tuberculous mycobacteria; TST, tuberculin skin test.

Table 3. QFT-GIT and TST results for 77 patients (data is available for 70 patients) suspected of TB infection and 44 patients (data is available for 40 patients) with confirmed TB disease

\begin{tabular}{cccccc}
\hline & & \multicolumn{3}{c}{ QFT-GIT } & Total \\
\cline { 3 - 4 } & & Negative & Positive & Indeterminate & \\
\hline \multirow{2}{*}{ TST, $n$} & Negative & $22 / 6$ & $7 / 6$ & $7 / 2$ & $36 / 14$ \\
& Positive & $10 / 8$ & $20 / 15$ & $4 / 3$ & $34 / 26$ \\
& Total & $32 / 14$ & $27 / 21$ & $11 / 5$ & $70 / 40$ \\
\hline
\end{tabular}

Data are presented as number of suspected TB results/confirmed TB results.

QFT-GIT: QuantiFERON-TB Gold In-Tube; TST: tuberculin skin test; TB: tuberculosis.

control group, whereas the result of TST was shown to be positive in 8 cases $(24.2 \%)$, and negative in 22 cases (66.7\%) (Table 3).

In total, 12 patients $(15.6 \%)$ showed indeterminate result on the QFT-GIT and all of them were due to positive control failure. They included 5 patients with TB, 3 patients with bacterial pneumonia, and 4 patients with fibrotic TB sequelae. Four patients among the non-TB group had the presence of previous TB scar, which indicated the possibility of LTBI. The median TB Ag-Nil and the median Mitogen-Nil were shown to be 0.015 (IQR, $-0.03 \sim 0.045)$ and 0.095 (IQR, $0.0825 \sim 0.275$ ) in the indeterminate group, of whom $58.3 \%$ (7 cases) showed negative TST results, and 33.3\% (3 patients with $\mathrm{TB}$ and 1 patient with non-TB group) showed positive
TST results. Among the 4 patients with positive TST results in indeterminate group, 1 patient had a previous history of TB treatment, and the patient was diagnosed with reactivated pulmonary $\mathrm{TB}$ at the time of colon cancer surgery. The other patient had active pulmonary TB with a history of BCG vaccination. Another patient died of respiratory failure caused by multidrug-resistant TB 20 days after mechanical ventilator management at the intensive care unit (ICU). Another patient in non-TB group who had a previous history of TB treatment had bipolar disorder and was hospitalized in the ICU for 15 days with severe sepsis.

The median age of the indeterminate group was shown to be 70.5 years (IQR, 55.5 76.0), which was significantly higher than that of the determinate group 
(51.0, IQR 41 66.0) $(\mathrm{p}=0.011)$. The age distribution of patients showing indeterminate results on QFT-GIT test is shown in Figure 1. Indeterminate results were significantly more frequent among the age bracket of 65 years or higher $(25.0 \%$ with indeterminate results vs. 18.4\% with determinate results) and age bracket of 75 years or higher $(33.3 \%$ vs. $7.6 \%)$. In the comparison of previous disease history, bronchial asthma (25.0\% vs. $3.1 \%, \mathrm{p}=0.004)$, hypertension $(58.3 \%$ vs. $21.5 \%, \mathrm{p}=$ $0.008)$, malignant disease $(25.0 \%$ vs. $4.6 \%, p=0.007)$, history of organ transplantation and steroid administration $(8.3 \%$ vs. $0 \%, p=0.019)$ were significantly high-

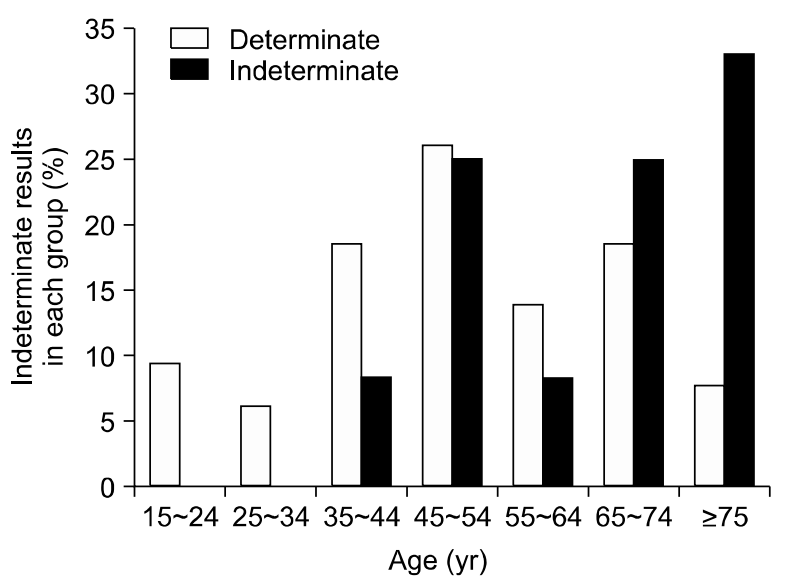

Figure 1. The proportion of determinate and indeterminate QFT-GIT results in each age group. QFT-GIT: QuantiFERON-TB Gold In-Tube. er in the indeterminate group than in the determinate group. The history of ICU stay was shown in 7 patients (58.3\%) within the indeterminate group, which was significantly higher than that of the determinate group $(18.5 \%, 12 / 65)(p=0.003)$. However, the median hospital stay of the indeterminate group was shown to be 15.0 (IQR, 5.0 40.0), which showed no difference with that of the determinate group (16.5; IQR, 7.0 38.0). The death rate of the indeterminate group was shown to be $25.0 \%$ (3 patients), which was higher than that of the determinate group $(7.7 \%, 5 / 65)$, but was not statistically significant $(p=0.071)$. In multivariate analysis, in the cases of age of 65 years or higher (odds ratio [OR], 11.29; $\mathrm{p}=0.026$ ), bronchial asthma (OR, 51.66; $\mathrm{p}=0.028)$, malignant diseases $(\mathrm{OR}, 5.90 ; \mathrm{p}=0.078)$ and history of ICU stay $(O R, 15.47 ; p=0.018)$, the frequency was shown to be higher in the indeterminate group than in the determinate group (Table 4).

In the blood tests, the lymphocytopenia $(0.7 ; \mathrm{IQR}$, $[0.4 \sim 1.1] \times 10^{3} / \mathrm{mm}^{3}$ vs. 1.3; IQR, [0.9 1.7] $\times 10^{3} / \mathrm{mm}^{3}$; $\mathrm{p}=0.013)$, particularly CD4 lymphocytopenia (147.0; IQR, $84.9 \sim 390.5 / \mathrm{mm}^{3}$ vs. 583.5; IQR, 332.3 849.0/ $\mathrm{mm}^{3}$; $\mathrm{p}=0.021)$, CRP elevation (2.7; IQR, $1.8 \sim 11.8 \mathrm{mg} / \mathrm{dL}$ vs. 1.2; IQR, 0.3 $5.0 \mathrm{mg} / \mathrm{dL} ; \mathrm{p}=0.014$ ), and hypoproteinemia (6.6; IQR, 5.9 6.8 g/dL vs. 7.2; IQR, 6.5 7.6 $\mathrm{g} / \mathrm{dL} ; \mathrm{p}=0.026)$ were significantly associated with indeterminate laboratory findings. However, no difference

Table 4. Multivariate logistic regression analysis of the indeterminate QFT-GIT results

\begin{tabular}{|c|c|c|c|c|}
\hline \multirow{2}{*}{ Clinical characteristics } & \multicolumn{2}{|c|}{ QFT-GIT test } & \multirow{2}{*}{ OR (95\% Cl) } & \multirow{2}{*}{$p$-value } \\
\hline & Indeterminate $(n=12,15.6 \%)$ & Determinate $(n=65,84.6 \%)$ & & \\
\hline \multicolumn{5}{|l|}{ Age, yr } \\
\hline$\geq 19$ and $<65$ & $5(6.5)$ & $47(61.0)$ & - & \\
\hline$\geq 65$ & $7(9.1)$ & $18(23.4)$ & $11.29(1.34 \sim 95.38)$ & 0.026 \\
\hline \multicolumn{5}{|l|}{ Underlying disease, \% } \\
\hline Asthma & $3(25.0)$ & $2(3.1)$ & $51.66(1.54 \sim 174.6)$ & 0.028 \\
\hline Hypertension & 7 (58.3) & $14(21.5)$ & $1.377(0131 \sim 14.45)$ & 0.790 \\
\hline Malignant disease & $3(25)$ & $3(4.6)$ & $5.901(0.370 \sim 94.04)$ & 0.078 \\
\hline Immunosuppressive treatment & $1(8.3)$ & $0(0)$ & ND & ND \\
\hline History of the ICU stay & 7 (58.3) & $12(18.5)$ & $15.47(1.59 \sim 150.92)$ & 0.018 \\
\hline
\end{tabular}

Data are presented as number (\%), unless otherwise indicated.

QFT-GIT: QuantiFERON-TB Gold In-Tube; OR: odds ratio; Cl: confidence interval; ND: not determined; ICU: intensive care unit. 
Tuberculosis and Respiratory Diseases Vol. 72. No. 5, May 2012

Table 5. Comparison of the results of TST and QTF-GIT test between 12 elderly patients and 32 younger patients within TB group

\begin{tabular}{lccrc}
\hline & Age $\geq 65$ yr $(\mathrm{n}=12)$ & Age $<65$ yr $(\mathrm{n}=32)$ & Total $(\mathrm{n}=44)$ & $\mathrm{p}$-value \\
\hline TST & & & & 0.019 \\
Positive & $4(36.4)$ & $22(75.8)$ & $26(65.0)$ & \\
$\quad$ Negative & $7(63.6)$ & $7(24.1)$ & & \\
QTF-GIT & $1(8.3)$ & $23(71.9)$ & $24(54.5)$ & 0.000 \\
Positive & $7(58.3)$ & $8(25.0)$ & $15(34.1)$ & \\
Negative & $4(33.3)$ & $1(3.1)$ & $5(11.4)$ & \\
Indeterminate & & & \\
\hline
\end{tabular}

Data are presented as number (\%).

TST: tuberculin skin test; QFT-GIT: QuantiFERON-TB Gold In-Tube; TB: tuberculosis.

in the radiologic finding and extension of lesion was found between the two groups.

In the TB group, comparisons among the group aged 65 years or higher (elderly patients, $n=12$ ) and the group aged less than 65 years (younger patients, $n=32$ ), no significant difference in the test results and demographic characteristics was found between the two groups. However, hypertension among underlying diseases was significantly higher in the elderly patients than in the younger patients $(\mathrm{p}=0.013)$. Thirty-six point four percent $(n=4)$ of the elderly patients were shown to be positive response in TST, whereas $78.5 \%(n=22)$ of the younger patients were shown to be positive response in TST $(p=0.019)$. In addition, the QFT-GIT positive rate was shown to be $54.5 \%(n=24)$ in the younger patients, but only $8.3 \%(\mathrm{n}=1)$ in the elderly patients, which was significantly lower in the elderly patients than in the younger patients $(p=0.000)$. The rate of the indeterminate $(33.3 \%, \mathrm{n}=4)$ and the false negative results $(58.3 \%, n=7)$ of QFT-GIT were higher in the elderly patients than in the younger patients (Table 5).

No lymphocytopenia $\left(<500 / \mathrm{mm}^{3}\right)$ was shown in 16 patients (36.4\%) who were false negative in QFT-GIT within the TB group. The 16 patients had 2 cases $(12.5 \%)$ of radiologically extensive lesion, 9 cases $(56.3 \%)$ of intermediate severity, and 5 cases (31.3\%) of mild severity. In addition, in the 21 patients (47.7\%) who were false negative or indeterminate in QFT-GIT within the TB group, the result of multivariate analysis showed that age of 65 years or higher (OR, 65.87; 95\% confidence interval [CI], 3.812 114.0; $\mathrm{p}=0.004)$ and history of ICU stay (OR, 41.90; 95\% CI, 2.33 752.4; $\mathrm{p}=0.011$ ) were risk factors.

\section{Discussion}

In this study, the result of QFT-GIT showed that $15.6 \%$ of the subjects were adjudged indeterminate due to positive control failure. The median age of the indeterminate group was 70.5 years, which was significantly higher than that of the determinate group (51.0 years). Indeterminate result was significantly higher in the patients with bronchial asthma and a history of ICU stay. In addition, the results of blood tests showed that the lymphocytopenia, including CD4 lymphocytopenia, CRP elevation, and hypoproteinemia were higher in the indeterminate group. The rate of indeterminate results of QFT-GIT was $11.4 \%$ in the TB group, and that of false negative results was $36.4 \%$, which was higher than that of TST $(31.8 \%)$. In the TB group aged 65 years or higher, the indeterminate results (33.3\%) and false negative results (58.3\%) accounted for 91.7\%, which showed that QFT-GIT alone had a limitation of being used for diagnosis of TB. According to the national statistics reported by the KNTA ${ }^{9}$, the rate of new TB cases for the past 10 years has been increasing. The rate of new TB cases of the elderly group aged 65 years of higher has been rapidly increas- 
ing from $11.5 \%$ in 2001 to $22.9 \%$ in 2004, and $27.2 \%$ in 2008. The possibility of the occurrence of TB is anticipated to increase in the elderly who have aging, various concurrent diseases, and resultant decrease in the immunologic status. In previous study in Taiwan, elderly patients with pulmonary TB were more likely to present with negative sputum smears and had more pleural effusion but fewer cavity findings on chest radiography. The prognosis is poor for the elderly TB patients with lower body weight, coexisting medical diseases, and extensive radiographic disease ${ }^{6}$. In other study in Korea, difference in AFB smear or culture positive rate between the elderly and the younger has not been observed'. However, it was suggested that the diagnostic yield of the elderly TB patients could be improved by applying methods such as the induced sputum using a saline solution nebulizer or bronchial washing fluid using bronchoscopy ${ }^{10}$. Potentially infectious or fatal patients should undergo diagnosis and treatment via clinical findings and radiologic examination, although they have negative results in sputum AFB smears or cultures. However, as it is not easy to diagnose and treat active TB in this case, NAA test of M.TB, pathologic examination, radiologic examination, TST and IGRAs are required. However, as the function of the $\mathrm{T}$ lymphocytes and amplification reaction decrease in the elderly patients ${ }^{6}$, the results of not only TST but also IGRAs should be carefully interpreted in elderly patients with TB.

For countries with high BCG vaccination rate and high TB morbidity, IGRAs rather than TST is particularly anticipated to be more helpful for diagnosis of TB infection. However, despite the usefulness of IGRAs in the diagnosis of LTBI, its usefulness in the diagnosis of active $\mathrm{TB}$ is still controversial ${ }^{11,12}$. A recently published meta analysis reported that the sensitivity of blood IGRAs was higher than that of TST for active TB, but its specificity was lower than that of TST, which showed a limitation in the discrimination between LTBI and active TB. The sensitivity and specificity of blood QFTGIT was reported to be $80 \%$ and $79 \%$, respectively. However, the indeterminate rate was also reported to reach $14.9 \%$ in adults ${ }^{1}$. QFT is considered limited if the result is indeterminate due to a difficulty in interpretation. As its frequency is higher than expected, the indeterminate result was reported to be $11 \%$ in a study conducted on healthy medical professionals ${ }^{13}$, most of whom were indeterminate due to positive control failure. Indeterminate result with positive control failure was associated with the decreased number and activity of $\mathrm{T}$ lymphocytes or the failure of IFN- $\gamma$ synthesis in $\mathrm{T}$ lymphocytes. In addition, the indeterminate result mainly occurred in patients with human immunodeficiency virus infection, malignant disease and renal dysfunction, which indicated the possibility of abnormal immune system ${ }^{13}$. Ferrara et al. ${ }^{14}$ and Kobashi et al. ${ }^{5}$ reported that $21 \%$ and $10.8 \%$ of patients who underwent QFT-G were indeterminate, respectively, and that in addition to receive the immunosuppressive drugs, age of 80 years or higher, lymphocytopenia, and malnutrition such as hypoproteinemia were the risk factors of the indeterminate results.

Because the QFT-GIT contains additional TB-specific antigens (TB7.7), it is speculated that the sensitivity is higher and indeterminate result is lower. However, consistent with the results of previous studies using the QFT-G test, the rate of indeterminate results was 15.6\% (12 cases) in the total subjects, and $11.4 \%$ (5 cases) in the $\mathrm{TB}$ group, which was lower than that of the non-TB group in our study. Indeterminate results were due to positive control failure in all 12 patients, and their median age was 70.5 years, which was significantly higher than that of the determinate group (51.0 years). In particular, no patient was adjudged indeterminate in the patients in their 40s or lower, and 67\% of the indeterminate group were patients in their 70 s or higher, showing a clear difference in the age bracket. The result of a Japanese study showed that no significant difference was found between TST (70\%) and QFT-G (87\%) in active TB patients aged less than 80 years, but that QFT positive rate $(76 \%)$ was significantly higher than TST positive rate $(27 \%)$ in active TB patients aged 80 years or higher. Thus, it was suggested that QFT could be more useful than TST if the number of lymphocytes 
is normal in the elderly patients ${ }^{15}$. In our study, however, both TST positive rate $(36.4 \%$ vs. $78.5 \%)$ and QFT-GIT positive rate $(8.3 \%$ vs. $54.5 \%)$ were significantly lower in the elderly patients aged 65 years or higher than in the patients aged less than 65 years within the TB group. In addition, the results of indeterminate and false negative rate in the elderly patients aged 65 years or higher were $33.3 \%$ and $58.3 \%$, respectively, which accounted that $91.7 \%$ in elderly patient was not diagnostic with QFT-GIT. Unlike indeterminate results due to positive control failure, QFT-GIT negative result refers to the cases of insufficient or no secretion of IFN from $\mathrm{T}$ cells that are stimulated by TB antigens despite its proper response to control antigens. It mainly occurs by the non-responsive state of cellular immune system, but may occur in the normal immune system. In addition, false negative result in QFT was reported to more occur in the cases of small amount of TB pathogen in the cultured sputum, small range of the lesion, and extrapulmonary TB such as TB pleurisy than in the case of lymphocytopenia ${ }^{15}$. But in our study, the patients with false negative QFT-GIT had more advanced disease (radiologically extensive to intermediate severity in 68.7\%) and most of whom were pulmonary TB except 2 miliary TB (13.3\%) and 1 TB pleurisy (6.7\%).

In previous studies reporting that the indeterminate rates of QFT-G and QFT-GIT varied from 0.1 to $40 \%$, patients receiving immunosuppressive drugs and immunocompromised patients were mainly included in the subjects ${ }^{7,14-19}$. Whereas, in this study, immunocompromised patients, patients receiving immunosuppressive drugs, and patients with end-stage renal disease accounted for only $7.8 \%$ of the total subjects, but the rate of indeterminate result was $15.6 \%$, which was not low. The main reason for this result is likely that our study population had many elderly and severely ill patients. The high indeterminate rate in the elderly patients is currently accepted to be associated with lymphocytopenia and malnutrition, as suggested by Kobashi et al. ${ }^{7}$. QFT test depends on inflammatory cytokines synthesized from $\mathrm{T}$ lymphocytes that are activated by TB-specific antigens. As the amount of IFN- $\gamma$ produced from the sensitized $\mathrm{T}$ cells is measured via ELISA in QFT test, IFN- $\gamma$ production is likely to decrease in the case of lymphocyte insufficiency ${ }^{18}$. In addition, Ferrara et al. ${ }^{14}$ reported that the rate of indeterminate result was higher in patients with the negative TST results, compared with the TST positive results (29\% vs. $7 \%$ ). In our study, the rate of indeterminate result was higher in the patients with negative TST results (19.4\%) than positive TST patients (11.8\%). In addition, 58.3\% of the indeterminate group had a history of ICU stay. In the cases of lymphocytopenia, particularly CD4 lymphocytopenia, CRP elevation, and hypoproteinemia in the blood tests were significantly associated with indeterminate results with the positive control failure. This result may also occur in patients with chronic disease and patients requiring ICU stay as well as in patients receiving immunosuppressive drugs. Thus, it is likely that difference in the immune responses of the elderly, chronic disease, and severely-ill patients could have affected the result. In addition, Kim et al. ${ }^{20}$ reported that the quantitative amount of IFN- $\gamma$ secretion and the number of $\mathrm{CD} 4+\mathrm{T}$ cells secreting IFN- $\gamma$ were inversely proportional to the severity of pulmonary $\mathrm{TB}$, which was consistent with the results of previous studies. They also reported that the limited sensitivity of QFT was shown in severe pulmonary TB due to the high indeterminate rate (19.0\%) in $\mathrm{QFT}^{20}$. In this study, however, due to the small number of TB patients within the indeterminate group, no correlation of indeterminate results with the extent of TB lesion was found.

We should consider the limitations of this study. Although a number of publications have demonstrated that excessive administration of blood samples to the tube may dilute IFN- $\gamma$ concentration, the survived $\mathrm{T}$ cells may be lost due to the delay after blood collection, and the rate of indeterminate result decreased by approximately $3 \%$ due to manual vortexing before cell culture $^{13}$, our study includes no information with variables of QFT-GIT. So, the increased rate of indeterminate result due to the errors of the test itself cannot be excluded. In addition, the time from blood collection 
to culturing was not checked in this study, despite the principle that blood collection should be conducted within 2 hours after delivery to the examination organization. Furthermore, although re-sampling is recommended to exclude the above test errors in the case of the positive control failure ${ }^{14}$, the re-sampling was not performed in this study. Thus, it is not clear whether non-responsive IFN- $\gamma$ was attributable to test errors, or it was a transient phenomenon, or non-responsive IFN$\gamma$ can be reversible with recovery from TB. Second, the numbers of the elderly patients with active TB and indeterminate group were insufficient to analyze within group. Third, BCG vaccination after birth is mandatory in Korea, and if the response to TST is negative at the time of senior in elementary school, BCG vaccination has been commonly administered again until 1997. However, it is uncertain to identify BCG vaccination status in a clinical practice, so we evaluated the status of vaccination with examination based on vaccination scar in some patients.

In this study, the sensitivity and specificity of QFTGIT were $52.3 \%$ and $57.6 \%$, respectively, which were lower than those of previous studies. That is, compared to the previous studies, the rates of false negative and indeterminate result were higher in this study. This is likely to be attributable to the higher proportion of severely-ill patients such as patients with chronic diseases and elderly patients, and patients requiring ICU stay and the differences in TB morbidity. In Korea, IGRAs are recommended for screening patients before immunosuppressive therapy with anti-tumor necrosis factor- $\alpha$ and for confirming TB infection in anyone who is TST-positive after contact with a patient with active pulmonary TB. However, IGRAs have not been recommended for use in the diagnosis of active TB. Nevertheless, based on previous study results, frequent use IGRAs in routine clinical practice suggesting a negative test result could rapidly exclude TB from a differential diagnosis $^{11}$. As it is, however, impossible to discriminate LTBI and active pulmonary TB via QFT in countries with a high burden of TB, the use of QFT test for the diagnosis of active pulmonary TB has a spe- cific concern in Korea with a modest morbidity rate of TB. In addition, in the cases of elderly patients, severely-ill patients requiring ICU stay, lymphocytopenia, CRP elevation and hypoproteinemia, indeterminate results that is not useful for diagnosis, or false negative results may be shown. Thus, a precaution should be given in the interpretation of the results. A multicenter IGRAs study, which is conducted on elderly Korean patients with active TB, is required, and a further study is also required to investigate the mechanism of indeterminate or false negative results.

\section{References}

1. Sester M, Sotgiu G, Lange C, Giehl C, Girardi E, Migliori $\mathrm{GB}$, et al. Interferon-gamma release assays for the diagnosis of active tuberculosis: a systematic review and meta-analysis. Eur Respir J 2011;37:100-11.

2. Lee HW, Park HY, Ahn YM, Sohn KC. Clinical significance of interferon gamma release assay for diagnosis of tuberculosis in children. Korean $\mathrm{J}$ Pediatr Infect Dis 2010;17:137-47.

3. Mazurek GH, Jereb J, Lobue $\mathrm{P}$, Iademarco MF, Metchock B, Vernon A, et al. Guidelines for using the QuantiFERON-TB Gold test for detecting Mycobacterium tuberculosis infection, United States. MMWR Recomm Rep 2005;54:49-55.

4. Mazurek GH, Jereb J, Vernon A, LoBue P, Goldberg $\mathrm{S}$, Castro K, et al. Updated guidelines for using interferon gamma release assays to detect Mycobacterium tuberculosis infection - United States, 2010. MMWR Recomm Rep 2010;59:1-25.

5. Kobashi Y, Sugiu T, Mouri K, Obase Y, Miyashita N, Oka M. Indeterminate results of QuantiFERON TB-2G test performed in routine clinical practice. Eur Respir J 2009;33:812-5.

6. Wang CS, Chen HC, Yang CJ, Wang WY, Chong IW, Hwang JJ, et al. The impact of age on the demographic, clinical, radiographic characteristics and treatment outcomes of pulmonary tuberculosis patients in Taiwan. Infection 2008;36:335-40.

7. Kobashi Y, Mouri K, Miyashita N, Okimoto N, Matsushima T, Kageoka T, et al. QuantiFERON TB-2G test for patients with active tuberculosis stratified by age groups. Scand J Infect Dis 2009;41:841-6.

8. Kang YA, Lee HW, Hwang SS, Um SW, Han SK, Shim YS, et al. Usefulness of whole-blood interferon-gamma 
assay and interferon-gamma enzyme-linked immunospot assay in the diagnosis of active pulmonary tuberculosis. Chest 2007;132:959-65.

9. Shin JY, Jung SY, Lee JE, Park JW, Yoo SJ, Park HS, et al. Characteristics of pulmonary tuberculosis in elderly people. Tuberc Respir Dis 2010;69:163-70.

10. Patel YR, Mehta JB, Harvill L, Gateley K. Flexible bronchoscopy as a diagnostic tool in the evaluation of pulmonary tuberculosis in an elderly population. J Am Geriatr Soc 1993;41:629-32.

11. Park SY, Jeon K, Um SW, Kwon OJ, Kang ES, Koh WJ. Clinical utility of the QuantiFERON-TB Gold In-Tube test for the diagnosis of active pulmonary tuberculosis. Scand J Infect Dis 2009; 41:818-22.

12. Park SY, Park YB, Choi JH, Lee JY, Kim JS, Mo EK. The diagnostic value of interferon-gamma assay in patients with active tuberculosis. Tuberc Respir Dis 2009; 66:13-9.

13. Miranda C, Yen-Lieberman B, Terpeluk P, Tomford JW, Gordon S. Reducing the rates of indeterminate results of the QuantiFERON-TB Gold In-Tube test during routine preemployment screening for latent tuberculosis infection among healthcare personnel. Infect Control Hosp Epidemiol 2009;30:296-8.

14. Ferrara G, Losi M, Meacci M, Meccugni B, Piro R, Roversi $\mathrm{P}$, et al. Routine hospital use of a new commercial whole blood interferon-gamma assay for the diagnosis of tuberculosis infection. Am J Respir Crit
Care Med 2005;172:631-5.

15. Kobashi Y, Mouri K, Yagi S, Obase Y, Miyashita N, Okimoto N, et al. Clinical utility of the QuantiFERON TB-2G test for elderly patients with active tuberculosis. Chest 2008;133:1196-202.

16. Kobashi Y, Fukuda M, Yoshida K, Oka M. An indeterminate QuantiFERON TB-2G response for miliary tuberculosis, due to severe pancytopenia. J Infect Chemother 2007;13:414-7.

17. Kobashi Y, Mouri K, Obase Y, Fukuda M, Miyashita N, Oka M. Clinical evaluation of QuantiFERON TB-2G test for immunocompromised patients. Eur Respir J 2007; 30:945-50.

18. Kobashi Y, Mouri K, Yagi S, Obase Y, Fukuda M, Miyashita N, et al. Usefulness of the QuantiFERON TB-2G test for the differential diagnosis of pulmonary tuberculosis. Intern Med 2008;47:237-43.

19. Kim EY, Lim JE, Jung JY, Son JY, Lee KJ, Yoon YW, et al. Performance of the tuberculin skin test and interferon-gamma release assay for detection of tuberculosis infection in immunocompromised patients in a BCGvaccinated population. BMC Infect Dis 2009;9:207.

20. Kim YY, Lee J, Lee YJ, Lee SY, Lee YH, Choi KJ, et al. Sensitivity of whole-blood interferon-gamma release assay according to the severity and the location of disease in patients with active tuberculosis. Tuberc Respir Dis 2011;70:125-31. 